\title{
On soft expert multiset theory
}

\author{
M. Gilbert Rani ${ }^{1 *}$, A. Gnana Arokiam² ${ }^{2}$ and K.Muthulakshmi ${ }^{3}$
}

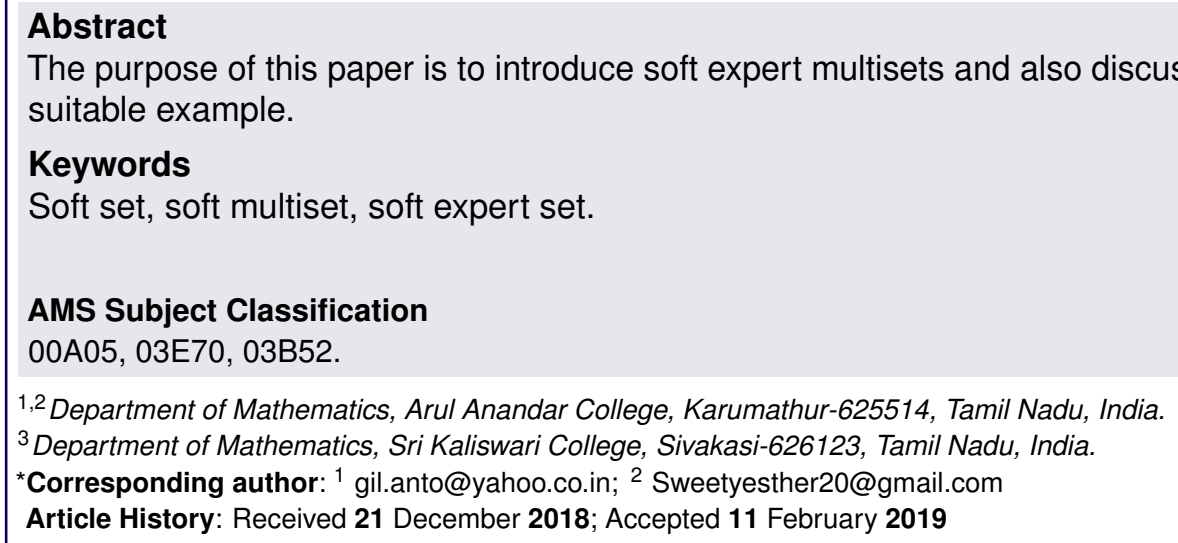

\section{Contents}

1 Introduction ................................5 57

2 Review ..................................... 57

3 Soft Expert Multiset ........................ 58

4 Application of soft expert multiset .............58

References ................................. 59

\section{Introduction}

The intricacy of the problem solving in medical science, engineering, social science, data mining, forensic science and so many fields, invited many sets to overcome such complexities and to tackle uncertainty and vagueness situations. Fuzzy set assigned membership value to the described attributes which help to organize all the information without any fail from information system. After the fuzzy set identification researchers paid their attention to this set to handle some peculiar situation occurred in given information system. Later, Pawlak[11] registered his own set, called rough set, which granulates the identical objects fro the information system in the name of similarity relation. The approximations are the bricks to find the rough sets. Many researchers found the relation between these two sets.

At the beginning of 20th century, Molodstov[9] acquainted his set, soft set, which is a function from the attribute set to the power set of an universal set. That attribute set carries non numerical values and the elements of universal set indicates some objects. Many research work done with these sets and the combination of the same sets. Soft set deals with one expert's opinion where as soft expert set talk about more than one expert opinion.

The collection of well defined objects, say "set", contains existence elements only not for their occurrences. Multiset[14] conceived the well defined elements with its multiplicity. That set helped to collect all the details about the elements from the information data.

In this paper we see the definition of soft expert multiset as well as its properties. The remaining subdivisions of this paper organized as follows in the following sequence section 2 recollects the needed definitions and notations of soft expert sets, section 3 introduces soft expert multiset, section 4 frames the applications of soft expert multiset.

\section{Review}

In this section, we make a review on basic ideas of this paper which will help to understand the forthcoming sections.

Throughout this paper, $U$ denotes a universal mset, $E$ indicates the set of all parameters. Let $P W(U)$ denote the set of all submset of $U$.

Definition 2.1. An mset $M$ drawn from the set $X$ is represented by function count $M$ or $C M$ defined as $C_{M}: X \rightarrow N$ where $N$ represents the set of non -negative integers. The word "multiset"often shortened to "mset".

Definition 2.2. Let $U$ be universal mset and $E$ be set of attributes. Then an ordered pair $(F, E)$ is called a soft multiset where $F$ is a mapping given by $F: A \rightarrow P W(U), P W(U)$ is defined as the set of all whole submset of $M$. 


\section{Soft Expert Multiset}

In this section let us introduce a new notion of soft expert set. Throughout this paper, let us denote $E$ is the set of all parameters and $X$ is a set of all experts. Let $O$ be a set of opinions from the experts, $Z=E \times X \times O$ and $A \subseteq Z$.

Definition 3.1. A Pair $\left(F_{M}, A\right)$ is called a soft expert multiset over $U$, where $F_{M}$ is a mapping constructed by

$$
F_{M}: A \rightarrow P W(U)
$$

In this paper, the opinion set $O$ conceives three values, That is, $O=\{0=$ disagree, $0.5=$ nocomments, $1=$ agree $\}$

Definition 3.2. Let $(F, A)$ and $(G, B)$ be soft expert multisets over $U .(F, A)$ is said to be a soft expert msubset of $(G, B)$ if

1. $A \subset B$.

2. for all $e \in B, G(e)$ is a msubset of $F(e)$.

This comparable relation can be denoted by $(F, A) \widetilde{\subseteq_{m}}(G, B)$.

Definition 3.3. The two soft expert multisets $(F, A)$ and $(G, B)$ over $U$ are said to be equal if $(F, A)$ is a soft expert msubset of $(G, B)$ and $(G, B)$ is a soft expert msubset of $(F, A)$.

Definition 3.4. Let $E$ be a set of parameters and $X$ a set of experts. The NOT set of $Z=E \times X \times O$ denoted by $Z^{m}=$ $\left\{\left(\grave{e}_{i}, x_{j}, o_{k}\right), \forall i, j, k\right\}$ where $\grave{e}_{i}$ is not $e_{i}$.

Definition 3.5. The complement of a soft expert mset $(F, A)$ is denoted by $(F, A)^{C}$ and is denoted by $(F, A)^{C}$ and is defined by $\left(F^{C}, \grave{A}\right)$ where $F^{C}: \grave{A} \rightarrow P(U)$ is a mapping given by $F^{C}(\grave{a})=$ $F^{r}(a)$ for every $\grave{a} \in \grave{A}$.

Definition 3.6. A soft expert msubset of $(F, A)$ is said to be 1-soft expert multiset $(F, A)_{1}$ over Udefined as follows:

$(F, A)_{1}=\left\{F_{1}(\alpha): \alpha \in E \times X \times\{1\}\right\}$.

Similarly we can define 0.5-soft expert multisetas well as 0 -soft expert multiset.

\section{Tabular Representation of soft expert multisets}

Let $U=\left\{m_{1} / h_{1}, m_{2} / h_{2}, \ldots \ldots \ldots \ldots \ldots m_{n} / h_{n}\right\}$ and $A=$ $\left\{e_{1}, e_{2}, \ldots \ldots, e_{m}\right\}$. We have three different tables for soft expert multisets, these are 1-soft expert multiset table,0.5-soft expert multiset table and 0-soft expert multiset table. The 1-soft expert multiset table can be formed as follows:

$$
\begin{aligned}
u_{i j} & =m_{i} \text { if } \mathrm{u}_{\mathrm{j}} \in\left(\mathrm{F}_{\mathrm{m}_{\mathrm{i}}}(\mathrm{ei})\right) \operatorname{andu}_{\mathrm{i}} \in \mathrm{F}_{1}(\alpha) \\
& =0 \text { otherwise }
\end{aligned}
$$

In this table $X_{1}, X_{2}, \ldots . . X_{n}$ denotes expert 1, expert $2, \ldots . .$. expert $n$. Like this table we can form the remaining two tables.

\begin{tabular}{lllll}
\hline & $u_{1}$ & $u_{2}$ & $\cdots$ & $u_{m}$ \\
\hline$\left(e_{1}, X_{1}\right)$ & $u_{11}$ & $u_{12}$ & $\cdots$ & $u_{1 m}$ \\
$\left(e_{2}, X_{2}\right)$ & $u_{21}$ & $u_{22}$ & $\cdots$ & $u_{2 m}$ \\
$\vdots$ & & $\vdots$ & & \\
$\left(e_{n}, X_{n}\right)$ & $u_{n_{1}}$ & $u_{n_{1}}$ & $\cdots$ & $u_{n m}$ \\
\hline
\end{tabular}

\section{Application of soft expert multiset}

Example 4.1. Suppose that a construction company wants to release a model for its model city making.

The model tells us how many houses are there?, how are they constructed? and so on. The promoters want to get a feed back from the experts in this field.

Let $U=\left\{4 / h_{1}, 5 / h_{2}, 3 / h_{3}, 8 / h_{4}, 7 / h_{5}, 4 / h_{6}, 3 / h_{7}, 5 / h_{8}\right\}$ be a set of all houses in their model city, $E=\left\{e_{1}, e_{2}, e_{3}, e_{4}\right\}$ be a set of decision parameters where $e_{1}$ indicates overall plan of this construction, $e_{2}$ indicates price, $e_{3}$ indicates different designs, $e_{4}$ indicates the facilities like water flow, transport, outlet of the unwanted things.

Let $X=\{r, s, t\}$ be a set of all experts. Suppose that the constructors have issued feed back form with some questions to the experts to file their opinions and make the rectification in that model if necessary.

$F\left(e_{1}, r, 1\right)=\left\{4 / h_{1}, 5 / h_{2}, 3 / h_{3}, 7 / h_{5}\right\}$,

$F\left(e_{1}, s, 1\right)=\left\{3 / h_{3}, 7 / h_{5}, 4 / h_{6}, 5 / h_{8}\right\}$,

$F\left(e_{1}, t, 1\right)=\left\{4 / h_{1}, 8 / h_{4}, 7 / h_{5}\right\}$,

$F\left(e_{2}, r, 1\right)=\left\{4 / h_{1}, 5 / h_{2}, 8 / h_{4}, 7 / h_{5}\right\}$,

$F\left(e_{2}, s, 1\right)=\left\{4 / h_{1}, 3 / h_{3}, 8 / h_{4}, 4 / h_{6}\right\}$,

$F\left(e_{2}, t, 1\right)=\left\{3 / h_{3}, 7 / h_{5}\right\}$,

$F\left(e_{3}, r, 1\right)=\left\{4 / h_{1}, 8 / h_{4}, 4 / h_{6}, 5 / h_{8}\right\}$,

$F\left(e_{3}, s, 1\right)=\left\{5 / h_{2}, 3 / h_{3}, 7 / h_{5}, 3 / h_{7}\right\}$,

$F\left(e_{3}, t, 1\right)=\left\{4 / h_{1}, 5 / h_{2}, 8 / h_{4}, 3 / h_{7}\right\}$,

$F\left(e_{4}, r, 1\right)=\left\{4 / h_{1}, 3 / h_{3}, 8 / h_{4},\right\}$

$F\left(e_{4}, s, 1\right)=\left\{4 / h_{1}, 8 / h_{4}, 3 / h_{7}\right\}$,

$F\left(e_{4}, t, 1\right)=\left\{4 / h_{1}, 7 / h_{5}, 3 / h_{7}\right\}$.

$F\left(e_{1}, r, 0.5\right)=\left\{8 / h_{4}, 3 / h_{3}, 3 / h_{7}\right\}$,

$F\left(e_{1}, s, 0.5\right)=\left\{4 / h_{1}, 8 / h_{4}\right\}$,

$F\left(e_{1}, t, 0.5\right)=\left\{5 / h_{2}, 3 / h_{3}, 3 / h_{7}\right\}$,

$F\left(e_{2}, r, 0.5\right)=\left\{3 / h_{3}, 4 / h_{6}\right\}$,

$F\left(e_{2}, s, 0.5\right)=\left\{5 / h_{2}, 7 / h_{5}\right\}, F\left(e_{2}, t, 0.5\right)=\left\{4 / h_{1}, 8 / h_{4}\right\}$,

$F\left(e_{3}, r, 0.5\right)=\left\{5 / h_{2}, 3 / h_{3}\right\}, F\left(e_{3}, s, 0.5\right)=\left\{4 / h_{1}, 8 / h_{4}\right\}$,

$F\left(e_{3}, t, 0.5\right)=\left\{3 / h_{3}, 7 / h_{5}\right\}, F\left(e_{4}, r, 0.5\right)=\left\{5 / h_{2}, 5 / h_{8}\right\}$,

$F\left(e_{4}, s, 0.5\right)=\left\{5 / h_{2}, 4 / h_{6}\right\}, F\left(e_{4}, t, 0.5\right)=\left\{5 / h_{2}, 3 / h_{3}\right\}$.

$F\left(e_{1}, r, 0\right)=\left\{4 / h_{6}, 5 / h_{8}\right\}, F\left(e_{1}, s, 0\right)=\left\{5 / h_{2}, 3 / h_{7}\right\}$,

$F\left(e_{1}, t, 0\right)=\left\{4 / h_{6}, 5 / h_{8}\right\}, F\left(e_{2}, r, 0\right)=\left\{3 / h_{7}, 5 / h_{8}\right\}$,

$F\left(e_{2}, s, 0\right)=\left\{3 / h_{7}, 5 / h_{8}\right\}$,

$F\left(e_{2}, t, 0\right)=\left\{5 / h_{2}, 4 / h_{6}, 3 / h_{7}\right\}$, 
$F\left(e_{3}, r, 0\right)=\left\{7 / h_{5}, 3 / h_{7}\right\}, F\left(e_{3}, s, 0\right)=\left\{4 / h_{6}, 5 / h_{8}\right\}$,

$F\left(e_{3}, s, 0\right)=\left\{4 / h_{6}, 5 / h_{8}\right\}$,

$F\left(e_{4}, r, 0\right)=\left\{7 / h_{5}, 4 / h_{6}, 3 / h_{7}\right\}$,

$F\left(e_{4}, s, 0\right)=\left\{3 / h_{3}, 7 / h_{5}, 5 / h_{8}\right\}$,

$F\left(e_{4}, s, 0\right)=\left\{7 / h_{5}, 4 / h_{6}, 5 / h_{8}\right\}$.

The following algorithm may be help to identify which house is better than other houses. After this finding the constructors will may develop their plans.

\section{ALGORITHM}

- input the soft multiset (F,A)

- find 1-soft expert multiset, 0.5-soft expert multiset and 0-soft expert multiset

- find $o_{i}=\sum_{i} u_{i j}$ for 1-soft expert multiset,

- find $v_{i}=\sum_{i} u_{i j}$ for 0.5-soft expert multiset,

- find $n_{i}=\sum_{i} u_{i j}$ for 0-soft expert multiset,

- find $d_{i}=o_{i}-\operatorname{maxv}_{i}, n_{i}$,

- find $h$, for which $d_{h}=\max \left\{d_{i}\right\}$

Now we proceed this algorithm with the above example. Table 1, table 2 and table 3 represent 1-soft expert multiset table, 0.5-soft expert multiset table and 0-soft expert multiset table respectively.

Table 1: 1-soft expert multiset table

\begin{tabular}{lllllllll}
\hline & $u_{1}$ & $u_{2}$ & $u_{3}$ & $u_{4}$ & $u_{5}$ & $u_{6}$ & $u_{7}$ & $u_{8}$ \\
\hline$\left(e_{1}, r\right)$ & 1 & 1 & 1 & 0 & 1 & 0 & 0 & 0 \\
$\left(e_{2}, r\right)$ & 1 & 1 & 0 & 1 & 1 & 0 & 0 & 0 \\
$\left(e_{3}, r\right)$ & 1 & 0 & 0 & 1 & 0 & 1 & 0 & 1 \\
$\left(e_{4}, r\right)$ & 1 & 0 & 1 & 1 & 0 & 0 & 0 & 0 \\
$\left(e_{1}, s\right)$ & 0 & 0 & 1 & 0 & 1 & 1 & 0 & 1 \\
$\left(e_{2}, s\right)$ & 1 & 0 & 1 & 1 & 0 & 1 & 0 & 0 \\
$\left(e_{3}, s\right)$ & 0 & 1 & 1 & 0 & 1 & 0 & 1 & 0 \\
$\left(e_{4}, s\right)$ & 1 & 0 & 0 & 1 & 0 & 0 & 1 & 0 \\
$\left(e_{1}, t\right)$ & 1 & 0 & 0 & 1 & 1 & 0 & 0 & 0 \\
$\left(e_{2}, t\right)$ & 0 & 0 & 1 & 0 & 1 & 0 & 0 & 0 \\
$\left(e_{3}, t\right)$ & 1 & 1 & 0 & 1 & 0 & 0 & 1 & 0 \\
$\left(e_{4}, t\right)$ & 1 & 0 & 0 & 0 & 1 & 0 & 1 & 0 \\
\hline$o_{i}=\sum_{i} u_{i j}$ & 9 & 4 & 6 & 7 & 7 & 3 & 4 & 2 \\
\hline
\end{tabular}

As per the experts recommendation $h_{1}$ type 4 houses that means $4 / \mathrm{h} 1$ are the best than other houses in this model city.
Table 1: 0.5-soft expert multiset table

\begin{tabular}{lllllllll}
\hline & $u_{1}$ & $u_{2}$ & $u_{3}$ & $u_{4}$ & $u_{5}$ & $u_{6}$ & $u_{7}$ & $u_{8}$ \\
\hline$\left(e_{1}, r\right)$ & 0 & 0 & 0 & 1 & 0 & 0 & 1 & 0 \\
$\left(e_{2}, r\right)$ & 0 & 0 & 1 & 0 & 0 & 1 & 0 & 0 \\
$\left(e_{3}, r\right)$ & 0 & 1 & 1 & 0 & 0 & 0 & 0 & 0 \\
$\left(e_{4}, r\right)$ & 0 & 1 & 0 & 0 & 0 & 0 & 0 & 1 \\
$\left(e_{1}, s\right)$ & 1 & 0 & 0 & 1 & 0 & 0 & 0 & 0 \\
$\left(e_{2}, s\right)$ & 0 & 1 & 0 & 0 & 1 & 0 & 0 & 0 \\
$\left(e_{3}, s\right)$ & 1 & 0 & 0 & 1 & 0 & 0 & 0 & 1 \\
$\left(e_{4}, s\right)$ & 0 & 1 & 0 & 0 & 0 & 1 & 0 & 0 \\
$\left(e_{1}, t\right)$ & 0 & 1 & 1 & 0 & 0 & 0 & 1 & 0 \\
$\left(e_{2}, t\right)$ & 1 & 0 & 0 & 1 & 0 & 0 & 0 & 0 \\
$\left(e_{3}, t\right)$ & 0 & 0 & 1 & 0 & 1 & 0 & 1 & 0 \\
$\left(e_{4}, t\right)$ & 0 & 1 & 1 & 0 & 0 & 0 & 0 & 0 \\
\hline$v_{i}=\sum_{i} u_{i j}$ & 3 & 6 & 5 & 4 & 2 & 2 & 2 & 2 \\
\hline
\end{tabular}

Table 1: 0-soft expert multiset table

\begin{tabular}{lllllllll}
\hline & $u_{1}$ & $u_{2}$ & $u_{3}$ & $u_{4}$ & $u_{5}$ & $u_{6}$ & $u_{7}$ & $u_{8}$ \\
\hline$\left(e_{1}, r\right)$ & 0 & 0 & 0 & 0 & 0 & 1 & 0 & 1 \\
$\left(e_{2}, r\right)$ & 0 & 0 & 0 & 0 & 0 & 0 & 1 & 1 \\
$\left(e_{3}, r\right)$ & 0 & 0 & 0 & 0 & 1 & 0 & 1 & 0 \\
$\left(e_{4}, r\right)$ & 0 & 0 & 0 & 0 & 1 & 1 & 1 & 0 \\
$\left(e_{1}, s\right)$ & 0 & 1 & 0 & 0 & 0 & 0 & 1 & 0 \\
$\left(e_{2}, s\right)$ & 0 & 0 & 0 & 0 & 0 & 0 & 1 & 1 \\
$\left(e_{3}, s\right)$ & 0 & 0 & 0 & 0 & 0 & 1 & 0 & 0 \\
$\left(e_{4}, s\right)$ & 0 & 0 & 1 & 0 & 1 & 0 & 0 & 1 \\
$\left(e_{1}, t\right)$ & 0 & 0 & 0 & 0 & 0 & 1 & 0 & 1 \\
$\left(e_{2}, t\right)$ & 0 & 1 & 0 & 0 & 0 & 1 & 1 & 1 \\
$\left(e_{3}, t\right)$ & 0 & 0 & 0 & 0 & 0 & 1 & 0 & 1 \\
$\left(e_{4}, t\right)$ & 0 & 0 & 0 & 0 & 1 & 1 & 0 & 1 \\
\hline$n_{i}=\sum_{i} u_{i j}$ & 0 & 2 & 1 & 1 & 4 & 7 & 6 & 8 \\
\hline
\end{tabular}

Decision Table

\begin{tabular}{llll}
\hline$o_{i}=\sum_{i} u_{i j}$ & $v_{i}=\sum_{i} u_{i j}$ & $n_{i}=\sum_{i} u_{i j}$ & $d_{i}=o_{i}-\max \left\{v_{i}, n_{i}\right\}$ \\
\hline$o_{1}=9$ & $v_{1}=3$ & $n_{1}=0$ & $d_{1}=6$ \\
$o_{2}=4$ & $v_{2}=6$ & $n_{2}=2$ & $d_{2}=-2$ \\
$o_{3}=6$ & $v_{3}=5$ & $n_{3}=1$ & $d_{3}=1$ \\
$o_{4}=7$ & $v_{4}=4$ & $n_{4}=1$ & $d_{4}=3$ \\
$o_{5}=7$ & $v_{5}=2$ & $n_{5}=4$ & $d_{5}=3$ \\
$o_{6}=3$ & $v_{6}=2$ & $n_{6}=7$ & $d_{6}=-4$ \\
$o_{7}=4$ & $v_{7}=2$ & $n_{7}=6$ & $d_{7}=-2$ \\
$o_{8}=2$ & $v_{8}=2$ & $n_{8}=8$ & $d_{8}=-6$ \\
\hline
\end{tabular}




\section{References}

[1] Cagman, N., Enginoglu, S.: soft set theory and uni-int decision making. European J. Oper. Res., 207(2010), 848855.

[2] Feng Feng, Changxing Li, Davvaz, B., Irfan Ali, M.: Soft sets combined with fuzzy sets and rough sets: a tentative approach, Soft Compute Springer14(2010), 899-911.

[3] Girish, K. P., John, S. J.: General relations between partially ordered multisets and their Chainsand antichains, Math. Commun., 14(2)(2009), 193-206.

[4] Gorzalzany, M. B.: A method of inference in approximate reasoning based on interval-valuedfuzzy sets, Fuzzy Sets and Systems, 21(1987), 1-17.

[5] Herawan, T., Mustafa, M. D.: On Milti-soft sets construction in in information systems, ICIC 2009 LNAi, Spring, Heidelberg, 5755(2009), 101-110.

[6] Jena, S. P., Ghosh, S. K., Tripathy, B. K.: On the theory of bags and lists, Inform. Sci., 132(2001), 241-254.

[7] P.K., Biswas, R., Roy, A.R.: Soft set theory,Math. Appl.,45(2003), 555-562.

[8] Maji, P.K., Roy, A.R.: An application of soft sets in a decision making problem, Comput. Math. Appl., 44(2002), 1077-1083.

[9] Molodtsov, D.A.: Soft set theory-first results,Comput. Math. Appl., 37(1999), 19-31.

[10] Ke Gong, Zhi Xiao, Xia Zhang, The bijective soft set with its operations, Computers and Mathematics with Applications, 60(2010), 2270-2278.

[11] Pawlak, Z.: Rough sets, Int. J. Inf. Comput. Sci., 1(1982), 341-356.

[12] SabirHussain, Bashir Ahmad: Some properties of soft topological spaces,Comput. Math. Appl., 62(2011), 40584067.

[13] Tanay, B., Kandemir, M.B.: Topological structure of fuzzy soft sets,Compute. Math. Appl.,61(2011), 2952 2957.

[14] Wayne D. Blizard: Multiset theory, Notre Dame J. Form. Log.,30(1989), 36-65.

[15] Won Keun Min: A note on soft topological spaces,Comput. Math. Appl., 62(2011), 3524-3528.

[16] Muhammad Irfan Ali: A note on soft sets, rough soft sets and fuzzy soft sets, A note on softsets, rough soft sets and fuzzy soft sets, Appl. Soft Comput. J. 12(2011), 23-32.

[17] LellisThivagar.M and Carmel Richard, On Nano Forms of Weakly Open sets,International Journal of Mathematics and Statistics Invention, 1(1)(2013), 31-37.

[18] Babitha K. V., Sunil J. J. On softmultiset, Annals of Fuzzy Mathematics and Informatics, 5(1)(2012), 35-44.

[19] K.P.Girish, Sunil Jacob John, On Multiset topologies, Theory and Applications of Mathematics and Computer Science, 2(1)(2012), 37-52.

[20] K.P.Girish, Sunil Jacob John, Rough multisets and its multiset topology, Transactions on Rough Sets XIV, LNCS 6600(2011), 62-80.

\author{
$\operatorname{ISSN}(\mathrm{P}): 2319-3786$ \\ Malaya Journal of Matematik \\ ISSN(O):2321 - 5666

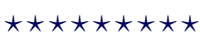

